Running Head: TRUST AND ATTENTION FOR MOTHER

Accepted for publication in "Journal of Experimental Child Psychology" Note: This is an uncorrected version of an author's manuscript accepted for publication. Copyediting, typesetting, and review of the resulting proofs will be undertaken on this manuscript before final publication. During production and pre-press, errors may be discovered that could affect the content.

Attachment-related attention bias plays a causal role on trust in maternal support

Guy Bosmans ${ }^{1}$, Alvaro Sanchez-Lopez ${ }^{2,3}$, Chloe Finet ${ }^{1}$, \& Rudi De Raedt ${ }^{2}$

1 Parenting and Special Education Research Group, KU Leuven, Belgium

2 Department of Experimental Clinical and Health Psychology, Ghent University, Belgium

3 Department of Clinical Psychology, Complutense University of Madrid, Spain

Corresponding author: Guy Bosmans, L. Vanderkelenstraat 32, 3000 Leuven, Belgium;

telephone: 0032163261 87; e-mail: guy.bosmans@kuleuven.be

Acknowledgement: This study was supported by FWO grants G077415N and G075718N and KULeuven grant C14/16/040 assigned to Guy Bosmans. The authors would like to thank Amit Lazarov and Yair Bar-Haim for their help during the design of the study. 


\begin{abstract}
The current study was designed to test whether children's ability to flexibly shift their attention (from mother during distress to peers during exploration, and vice versa) causally increases children's trust in their mothers' support. We trained attention flexibility using a gaze-contingent music-reward design. Eighty-five children (46\% boys; aged 9 to 13 years), were randomly assigned to this training or a comparable yoked-control condition. Attentional preferences were measured via eye-tracking. Before and after the manipulation we measured self-reported trust. Results showed that the training condition increased children's attention flexibility. Training-related increased attentional focus on mother during distress was linked with increased trust.
\end{abstract}




\section{Attachment-related attention bias plays a causal role on trust in maternal support}

Accumulating attachment research shows that preferences in the processing of attachment-related information are linked to children's trust in maternal support during stress (Dykas \& Cassidy, 2011). Different levels of trust have been related to differences or biases in attention to, recollection of, and interpretation of more positive versus negative attachment-related information (Kirsh \& Cassidy, 1991; Zimmermann \& Iwanski, 2015). These biases are supposed to serve stability in attachment-related expectations (Bowlby, 1969). However, this assumption has remained largely untested, creating a significant gap in our understanding of attachment and attachment development (e.g., Thompson, 2008). De Winter, Bosmans and Salemink (2017) preliminarily supported this assumption in middle childhood by showing that training children to interpret maternal behavior as more supportive increased their self-reported trust. Although this study was important as the first research evidence for causal effects of information processing biases on trust levels, interpretation bias training and self-reported trust rely on similar explicit verbal evaluation strategies. Consequently, this raises the concern that De Winter et al.'s (2017) effects might merely reflect a methodological overlap between the training and the outcome measure. A more stringent test of Bowlby's assumption would involve finding causal effects of attachment-related attention biases on children's trust in maternal support.

Attentional processes can be considered the first and, therefore, most basic stage of information processing (Derryberry \& Tucker, 1994), and they have been shown to determine how information is subsequently elaborated and interpreted (e.g., Sanchez, Everaert, De Putter, Mueller \& Koster, 2015). Therefore, numerous studies have been conducted aimed at changing individuals' attentional preferences using Attention Bias Modification (ABM; e.g., Heeren et al., 2015). Standard ABM techniques aim at training 
attention toward positive stimuli and/or away from negative stimuli. The idea behind this procedure is that a certain cognition (e.g., fear of negative social feedback) will decrease if pre-existing negative attention biases (i.e., preferential attention to negative social feedback) are changed for more positive attention biases (i.e., training to redirect attentional focus toward alternative neutral or positive social feedback). Yet, despite the theoretical potential of this approach, current meta-analyses suggest that these standard ABM trainings yield at best small effects (e.g., Cristea, Kok, \& Cuijpers, 2015; Heeren et al., 2015).

One reason for the small effect sizes might be that the function of attention bias has not been conceptualized optimally in prior studies. For the current study, we conjectured that one alternative perspective on attention biases might be that these biases (e.g. toward positive or negative social feedback) are not adaptive or maladaptive per se, but adaptive to the extent that attentional focus can vary flexibly in response to the individuals' contextual demands. Particularly in the context of middle childhood attachment, recent research would support this proposal. For example, Bosmans et al. (2017) showed that children have an attentional preference for their mother, but whether this preference is adaptive or maladaptive depends on factors like children's stress and trust levels (e.g., Van de Walle et al., 2017). Children with more trust seem protected in stressful situations if they are more strongly focused on their mother, but the same attentional focus on mother becomes a liability if children lack trust (e.g, Bosmans et al., 2013). These findings raised the hypothesis that, instead of being an adaptive or maladaptive trait, attentional preference for the mother might flexibly adjust to whether or not children momentarily need their mother's proximity and support. 
The relevance of attachment-related attention flexibility was previously proposed by Main (2000) and Betherton (1985), who argued that children with more trust should be able to shift their attention focus to their mother during stress and to exploration-relevant information (e.g., to explore how rewarding positive interactions with peers can be) in absence of stress. Children with less trust are supposed to struggle to find a good attachment-exploration balance (Main, Hesse, \& Kaplan, 2005). Instead, their attentional strategy is assumed to depend on whether they are more anxiously or more avoidantly attached. On the one hand, more anxiously attached children seek maternal support during distress but constantly fear rejection and disappointment, which is supposedly reflected in attentional preference for their mother at the expense of exploration (Cassidy, 1994). On the other hand, more avoidantly attached children refuse support seeking and avoid close contacts with caregivers, which is supposedly reflected in an attentional preference for exploration at the expense of proximity with the mother (Cassidy, 1994). Although Main's (2000) theory is generally accepted within the field of attachment theory, so far, this theory has not been directly tested.

Moreover, Main's theory did not consider the likelihood that these biases may play a causal role in trust development and maintenance. In line with Bowlby's (1969) prediction that these biases serve stability in attachment-related expectations, an accumulating number of studies suggest that expectations are the consequence rather than the cause of information processing biases (e.g., MacLeod et al., 2009). According to this research, the brain filters information from the environment in such a way that information more likely gets processed and encoded when that information is congruent with previous experiences. This information subsequently affects the appraisals of the environment and eventually the content of the related expectations (Baert et al., 2011). In line with this line of reasoning, 
recent research has shown that training children to interpret maternal behavior as more supportive increased their self-reported trust (De Winter, Bosmans, \& Salemink, 2017) and improved access to positive attachment-related memories (De Winter, Salemink, \& Bosmans, 2018).

Following this logic, the best way to test Main's attention flexibility hypothesis would be to manipulate children's attachment-related attention flexibility and then see whether this manipulation changes the attachment-related expectations. In other words, if children's ability to flexibly shift their attention from mother to other stimuli depending on the context is indeed a relevant feature of secure attachment (Main, 2000), the best way to evaluate this association is to test whether changes in attention flexibility explain changes in children's attachment-related expectations. This would require to measure attachment-related expectations before the experiment, then manipulate children's attention flexibility, and subsequently measure the attachment-related expectations again.

To manipulate children's attention flexibility, we designed an Attention Flexibility Training (AFT) during which two types of trials are presented. We focused on the attentional processing of the mother and of peer interactions because peers are relevant both as a stressor and as a source for exploration in middle childhood (Vandevivere et al., 2015). Stress trials consisted of pictures of children's own mother and pictures of bullying peers, whereas exploration trials consisted of the same mother pictures and pictures of positive interactions with peers. To train attentional flexibility, we used a gaze-contingent music reward design (Lazarov, Pine, \& Bar-Haim, 2017). Using eye-tracking technology, children in the training condition were rewarded with music when they looked at the mother pictures in the stress trials and when they looked at the peer pictures in the exploration trials. In the 
yoked-control condition, music started and stopped playing in a similar pattern as the training condition, but not contingently on their attentional behavior.

To assess change in attention flexibility throughout the procedure we first assessed children's focus on their mother versus their peers during distressing versus exploration contexts. For this purpose, we measured for each context the duration of children's fixation on their mother or their peers and the number of times children fixated on both stimuli. As a second indicator of attentional flexibility, we assessed children's switching cost. Switching cost refers to the extent to which children display difficulties to change between attentional processing strategies. Low switching cost refers to children who can quickly start exploring (focusing on the peers) in the exploration trials, but can quickly shift their attentional strategy to support seeking (focusing on the mother) during the stress trials, an vice versa. High switching cost suggests that children have difficulties to switch between both attentional processing strategies (i.e., low attention flexibility).

Using this paradigm, we tested whether trust levels were causally affected by training children to flexibly switch their attentional focus from their mother to exploration-relevant stimuli depending on the level of distress in the context. Nevertheless, Main's (2000) hypothesis could also imply that children's attachment anxiety or attachment avoidance reduces the effectiveness of such a training, both on changes in the attentional flexibility as well as on changes in trust. Therefore, we additionally tested whether attachment anxiety and attachment avoidance moderate the outcome of the AFT. We tested these hypotheses in middle childhood, because prior research has shown that this is a relevant age-group to study attachment-related developmental processes (De Winter et al., 2017).

In sum, in the current study we tested first the prediction that AFT increases attention flexibility. Specifically, we expected that children in the AFT condition would 
become more focused on their mother during the stress trials, more focused on peers during the exploration trials, and show decreases in their switching costs at the end of the training procedure. Second, we predicted that training these attention flexibility patterns increases children's self-reported trust. We tested this prediction in two ways. On the one hand, we compared training versus yoked-control children's change in trust before versus after the procedure. On the other hand, we tested whether training-induced changes in attention were correlated with changes in trust. Furthermore, we tested whether the association between the training and changes in trust were indirectly linked through training-induced changes in attention. Finally, we repeated all the analyses and included attachment anxiety and attachment avoidance as potential moderators of the manipulation outcomes.

\section{Method}

\section{Participants}

In total 85 children ( $46 \%$ boys) with ages ranging from 9 to 13 years $(M=10.7, S D=$ .86) were randomly assigned to a training $(n=43)$ or a yoked-control $(n=43)$ condition. Participating children lived together with their biological mother $(n=84)$ or adoptive mother $(n=1)$ and lived in $74.1 \%$ of the cases together with both parents.

\section{Procedure}

Flyers were distributed in schools to invite children to participate. When parents gave their informed consent, children were tested individually in schools. During each session, the procedure was explained, and children gave informed assent. At the start of the study, we administered pre-training questionnaires comprising measures of trust, and attachment anxiety and avoidance. Then, they were randomly assigned to either the AFT or the yokedcontrol condition. Finally, we administered post training measures of trust, and attachment 
anxiety and avoidance. The entire procedure was approved by the local university's ethical committee.

\section{Measures}

Attention Flexibility Training (AFT) paradigm. Eye movements were monitored via a TOBII X2-60 Compact eye tracker while participants completed the AFT procedure. Visual fixations were defined as a minimum duration of $100 \mathrm{~ms}$ and a maximum fixation radius of 1 degree.

Procedure. The training condition aimed to increase children's ability to flexibly switch their attention from orientation toward the mother in a distress context (bullying) to orientation toward exploration in a non-distressing context (positive peer interactions). For this purpose, we designed a training comprised of two trial types. In the stress trials, children were presented with 4 pictures of their own mother and 4 pictures referring to bullying contexts (see Figure 1). In the exploration trials, next to the 4 mother pictures, 4 pictures referring to positive peer interaction contexts were presented (see Figure 2). To train children's attention flexibility across these different contexts, we adapted Lazarov et al.'s (2017) gaze-contingent music-reward paradigm. Before the start of the experiment, children were given the opportunity to select their favorite songs. Then, we used the selected songs to reward children when they looked (i.e., made a visual fixation) at the corresponding training-relevant targets in each type of context. Therefore, in the training condition, music was played contingent upon children's visual fixations on the target stimulus (mother in the stress trials; peers in the exploration trials: i.e., contingent to detection of a visual fixation on the corresponding stimulus type by the eye-tracker). In the yoked-control condition, music patterns (whole duration across the trial, number of pauses across the trial) were programmed to play identically to the music patterns recorded for children in the training 
condition, but with playing times not being contingent to children's visual fixations. This was based on the average duration-pauses of a first subgroup of children in the training condition for each trial $(n=12)$. Therefore, children in the control condition received the same type of reinforcement as children in the training group (i.e., same amount of music, same pause-play patterns), but music was, by design, no longer $100 \%$ contingent upon children's gaze. Instead, music played at random predefined periods, independently from children's visual fixations. The full training was composed of 32 trials (16 stress, 16 exploration trials), with each trial lasting 22 seconds. The positions of the pictures were randomly distributed over trials and trial type. The order of the trial types was pseudorandomized, in order to obtain indices of switching cost changes across the training (see further explanation below).

Dependent variables. First, to evaluate the effect of the training on children's attention bias on the mother versus peers during the two trial types, we focused on two types of eye-tracking indices that were recorded throughout the whole duration of a trial: total fixation duration (the total time children fixated on the mother or peers during the trial) and fixation count (how often children fixated their gaze on the mother or peers during the trial). We calculated the total fixation duration and the fixation count on the mothers' and peers' areas of interest during stress and during exploration trials separately. To evaluate training effects on these attention bias measures, we compared children's performance on each of the two types of indices in each type of context at the start of the training (average across the first 8 trials of training) to the performance on these indices at the end of the training (average across the last 8 trials of training).

Second, to evaluate the effect of the training on children's ability to switch attention across contexts, we computed children's attention switch cost, which refers to children's 
ability to flexibly switch attention from/to the mother during stress trials to/from peers during exploration trials. This index was based on the time children needed to made a first fixation on the target mother versus peers, depending on the context. For this purpose, we programmed both repetition trials (two subsequent trials with the same context and task: i.e., two exploration/attend peers-trials or two stress/attend mother-trials, presented consecutively) and alternation trials (two subsequent trials with a different context and task: i.e., an exploration/attend peers-trial followed by a stress/attend mother-trials, or a stress/attend mother-trial followed by an exploration/attend peers-trial). Switch cost indices were determined by the difference between the time to first fixate the target stimulus in repetition vs. alternation trials. To evaluate training effects on switch cost, we compared switch cost scores at the start of the training to switch cost scores at the end of the training. To ensure there were enough trials to calculate switch cost scores, we compared the first versus the second half of the trials. Negative scores suggest switch cost (i.e., longer times to fixate the target stimuli when alternation is required according to the context-rule), scores around 0 suggest absence of switch cost, whereas positive scores suggest that children are more inclined toward switching compared to maintaining attentional focus. Because the training effects were similar for both switch cost from exploration to stress trials and from stress to exploration trials, we calculated general switch cost factor scores across both indices for each half of the experiment (factor loadings for each index were higher than .70).

Self-reported measures. Trust in maternal support was measured with the trust subscale of the People in My Life questionnaire (Ridenour et al., 2006). This measure has been frequently used before in middle childhood attachment research and has proven its reliability and validity in a host of studies (e.g., De Winter et al., 2017). The measure consists of 10 items (e.g., "I can count on my mother to help me when I have a problem") and are 
scored on a 4-point Likert scale, ranging from 1 (almost never true) to 4 (always true). In the current study, internal consistency was good, $\alpha_{\text {pre }}=.72, \alpha_{\text {post }}=.75$.

Attachment Anxiety and Avoidance was measured with the middle childhood short version of the Experiences of Close Relationships - Revised questionnaire in which all 12 items focused on the relationship with the mother (Brenning et al., 2014). Attachment anxiety is measured with 6 items (e.g. "I'm worried that my mother might want to leave me.") just like Attachment avoidance (e.g. "I prefer not to get too close to my mother."). The items are scored on a 7-point Likert scale ranging from 1 (strongly disagree) to 7 (strongly agree). The validity of the measure has been established with observation research (Dujardin et al., 2016). The measure's internal consistency was good both before $\left(\alpha_{\text {anxiety }}=.88\right.$, $\left.\alpha_{\text {avoidance }}=.73\right)$ and after the training $\left(\alpha_{\text {anxiety }}=.90, \alpha_{\text {avoidance }}=.73\right)$.

\section{Plan of the analyses}

To test whether the training indeed changed children's attentional inclination to approach the mother (in stress trials) versus approach peers (in exploration trials) across the procedure, we conducted two 2 (between-subjects Condition: AFT versus yoked control) X 2 (within-subjects Time: start versus end of the training) $\times 2$ (within-subjects Trial Type: stress versus exploration) $\times 2$ (within-subjects Target Stimulus: mother versus peer pictures) mixed ANOVAs with total fixation duration and fixation count as dependent variables respectively. In addition, to test training effects on the switch cost, we conducted a 2 (between-subject Condition: AFT versus control) $\times 2$ (within-subject Time: first versus second half of the training) mixed ANOVA with switch cost scores as dependent variable. To assess the effect sizes, we calculated $\eta_{p}{ }^{2}$ for the F-tests. In the follow-up analyses, we calculated Cohen's $\mathrm{d}_{\mathrm{av}}$ for the $t$-tests. Cohen's $d_{a v}$ is a conservative but better assessment of effect size than Cohen's $d_{z}$, with the same criteria (Lakens, 2013). 
To test training effects on trust, we first conducted a 2 (between-subject Condition: AFT versus control) $\times 2$ (within-subject Time: pre versus post) mixed ANOVAs with trust as dependent variable. Second, we calculated correlations between changes in self-reported attachment and changes in attentional preference in the stress and exploration conditions separately. More specifically, we conducted bivariate correlations between measures of changes in attention and in trust. Residualized change scores were constructed using simple linear regression models (Segal et al., 2006), where the initial measure of the corresponding variable (e.g., pre-training trust level, attention duration to mother in the first stress trials) were entered in the regression model as predictors of the final measure of that variable (e.g., post-training trust level, attention duration to mother in the last stress trials), and the resulting standardized residuals served as measures of change across the training in the corresponding variable. A standardized residual change score expresses the extent to which individuals' scores deviate from their expected value because they more strongly increased (scores above 0) or because they more strongly decreased (scores below 0 ).

To investigate whether the training condition (dummy coded AFT versus yokedcontrol) indirectly led to changes in trust via its transfer effects in attentional preferences and attentional switching capacity (i.e., training condition $\rightarrow$ attention change across the training $\rightarrow$ pre-post trust change), we conducted mediation analyses. For this purpose, we used a nonparametric resampling method (bias-corrected bootstrap) with 10,000 resamples drawn with replacement from the original sample $(n=83)$ to derive the $95 \%$ confidence interval (CI) for the indirect effect. Analyses were conducted with PROCESS (Hayes, 2012).

Finally, to test the moderating effects of attachment anxiety and attachment avoidance on the manipulation effects, all the above-mentioned main analyses were repeated with both insecure attachment scales as moderator. For the repeated measures 
mixed ANOVA analyses, attachment anxiety/avoidance was added as a covariate that was defined as a moderator of the condition effects. For the correlation analyses, we conducted two-way interaction hierarchical regression analyses in PROCESS. For the indirect effect analysis, we conducted a moderated mediation analysis in PROCESS.

\section{Results}

\section{Preliminary analyses}

Overall, less than $2 \%$ of the data were missing. Missing data were list wise deleted. Both condition groups were equal regarding age, $F(1,84)=.08, p=.773$ and the attachment measures before the experiment: trust, $F(1,83)=2.28, p=.135$, attachment anxiety, $F(1,80)$ $=.17, p=.685$, and attachment avoidance, $F(1,80)=2.18, p=.144$. Quality of the eyetracking recordings was excellent as reflected in the percentage of accuracy (i.e., percentage of times that the position of both eyes was successfully estimated by the eye-tracker, $M=$ $91.60 \% ; S D=6.12 ;$ minimum value $=72 \% ;$ maximum value $=99 \%$.

\section{Attention preference training evaluation}

\section{Total fixation duration training effects.}

Table 1 shows a significant four-way interaction. To further probe the interaction effect, 2 (Condition) X 2 (Time) X 2 (Target Stimulus) repeated measures ANOVAs were conducted separately for each Trial Type (exploration versus stress trials). Analysis of the exploration trials showed no significant Condition X Time X Target Stimulus interaction, $F(1,82)=1.09, p=.300, \eta_{p}^{2}=.01$. This result suggests that the training did not produce significant changes in attention to peers vs. mother over time during exploration trials. This result appears to be mainly due to a strong Target Stimulus main effect, $F(1,83)=53.21, p<$ $.001, \eta_{p}^{2}=.39$. Across the training, all children fixated more on peers, $M=9.30, S D=3.06$, compared to mother, $M=5.39, S D=2.66$, during exploration trials. Analysis of the stress 
trials, instead, showed a significant Condition $X$ Time $X$ Target Stimulus interaction, $F(1,82)=$ 25.63, $p<.001, \eta_{p}{ }^{2}=.24$. This result suggests that AFT had an effect at the level of the stress trials. Figure 3 shows the stress trial effects. In line with the AFT goal, during stress trials, children receiving AFT showed increased attention preferences for the mother, $t(42)=3.05$, $p<.01, d_{a v}=.61$, and decreased attention preferences for peers across time, $t(42)=5.01, p<$ $.001, d_{a v}=.81$. However, in the yoked control condition there was a decreased attention preference for the mother, $t(40)=3.41, p<.001, d_{a v}=.37$, and an increased attention preference for peers across time, $t(40)=2.48, p<.05, d_{a v}=.33$.

\section{Number of fixations training effects}

Table 1 shows a significant four-way interaction. To further probe the interaction effect, 2 (Condition) X 2 (Time) X 2 (Target Stimulus) repeated measures ANOVAs were conducted separately for each Trial Type (exploration versus stress trials). Analysis of the exploration trials showed no significant Condition X Time X Target Stimulus interaction, $F(1,82)=.79, p=.378, \eta_{p}{ }^{2}=.01$. There was, however, again a main effect of Target Stimulus, $F(1,83)=258.23, p<.001, \eta_{p}{ }^{2}=.76$, showing a general attention preference for peers, $M=$ $41.75, S D=14.96$, compared to mother, $M=23.11, S D=11.03$, across trials. Analysis of the stress trials, again, showed a significant Condition X Time X Target Stimulus interaction, $F(1,82)=30.94, p<.001, \eta_{p}^{2}=.27$. This finding suggests that AFT had an effect at the level of the stress trials. Figure 4 shows the stress trial effects. In line with the AFT goal, during stress trials, the training resulted in increased attention preference for the mother, $t(42)=2.14, p<$ $.05, d_{a v}=.47$, and decreased attention preference for peers across time, $t(42)=4.61, p<$ $.001, d_{a v}=.59$. However, in the yoked control condition there was a decreased attention preference for the mother, $t(40)=4.09, p<.001, d_{a v}=.51$, and an increased attention preference for peers, $t(40)=1.85, p=.071, d_{a v}=.33$. 


\section{Switch cost training effects}

The Condition X Time interaction was significant, $F(2,81)=42.84, p<.001, \eta_{p}^{2}=.34$ (Figure 5). Further probing the interaction effect showed that the switch cost became more negative (i.e., larger switching costs) in the control condition, $t(40)=5,51, p<.001, d_{a v}=$ 1.08, and more positive in the ABM training condition (i.e., larger facilitated switching capacity) $, t(42)=4,07, p<.001, d_{a v}=.80$, across the training procedure. At the end of the training, children in the ABM training condition had a significantly higher score (i.e., higher attentional switching capacity) compared to children in the yoked-control condition, $F(1,83)$ $=59,60, p<.001, d_{a v}=1.68$.

\section{Training effects on trust}

First, no condition $\mathrm{X}$ time interaction effects on trust, $F(1,82)=1,97, p=.164, \eta_{p}{ }^{2}=$ .02 was found. Second, because the above-mentioned analyses showed that all training effects occurred in the stress trials, the correlation analyses focused on changes in trust and in attentional biases toward the mother and peers in the stress trials. Table 2 shows that only in the training condition pre-post increases in trust were significantly linked with increased focus on the mother (significantly for fixation count and marginally significantly for total fixation duration) and with decreased focus on peers (significantly for total fixation duration) across the training.

\section{Indirect effects}

Results showed that both changes in mother-related total fixation duration and fixation count indirectly linked the AFT condition to changes in trust. The AFT condition significantly increased the attentional preference for the mother (expressed in unstandardized regression weights: for fixation duration, $b=.92, S E=.20, p<.001$; for fixation count, $b=.83, S E=.20, p<.001$ ) which was significantly linked to increases in trust 
(for fixation duration, $b=.25, S E=.12, p<.05$; for fixation count, $b=.27, S E=.12, p<.05$ ). The indirect effect of fixation duration, $b=.23, S E=.11$, was estimated to lie between .05 and .49. The indirect effect of fixation count, $b=.22$, $S E=.08$, was estimated to lie between .09 and .4 . Because zero is not in the $95 \% \mathrm{Cls}$, the indirect effects are significantly different from zero at $p<.05$ (two tailed). No significant indirect effects were found for changes in attention focus on peers, nor for switch cost change.

\section{Moderation analyses}

For the changes in fixation duration, the Condition X Time X Trial Type X Target Stimulus $X$ attachment anxiety mixed ANOVA was not significant, $F(1,76)=.373, p=.543$, $\eta_{p}{ }^{2}=.01$. Also, the Condition X Time X Trial Type X Target Stimulus X attachment avoidance repeated measures mixed ANOVA was not significant, $F(1,76)=.679, p=.413, \eta_{p}{ }^{2}=.01$. For the changes in fixation counts, the Condition X Time X Trial Type X Target Stimulus X attachment anxiety repeated measures mixed ANOVA was not significant, $F(1,76)=2.26, p=$ .137, $\eta_{p}^{2}=.03$. Also, the Condition X Time X Trial Type X Target Stimulus X attachment avoidance repeated measures mixed ANOVA was not significant, $F(1,76)=.607, p=.438, \eta_{p}^{2}$ $=.01$. For the changes in switching cost, the Condition $X$ Time $X$ attachment anxiety repeated measures mixed ANOVA was not significant, $F(1,76)=.573, p=.451, \eta_{p}^{2}=.01$. Also, the Condition $\mathrm{X}$ Time $\mathrm{X}$ attachment avoidance repeated measures mixed ANOVA was not significant, $F(1,76)=.032, p=.859, \eta_{p}^{2}=.01$. For the changes in trust, the Condition X Time $X$ attachment anxiety repeated measures mixed ANOVA was not significant, $F(1,76)=.754, p$ $=.388, \eta_{p}^{2}=.01$. Also, the Condition $\mathrm{X}$ Time $\mathrm{X}$ attachment avoidance repeated measures mixed ANOVA was not significant, $F(1,76)=3.44, p=.067, \eta_{p}^{2}=.04$ 
In addition, in the stress trials, the associations between changes in trust and changes in the attentional processing indices were not moderated by both anxious and avoidant attachment, $p$-values $>.449$. Finally, the indirect effect of condition on changes in trust through changes in fixation duration on mother were not moderated by attachment anxiety, $b=.01, p=.795$, nor by attachment avoidance, $b=-.03, p=.271$. Also, the indirect effect of condition on changes in trust through changes in fixation count on mother were not moderated by attachment anxiety, $b=-.03, p=.255$, nor by attachment avoidance, $b=-.03$, $p=.392$.

\section{Discussion}

In the current study, we tested whether biases in children's attentional processing of attachment-related information have a causal impact on children's trust in maternal support. We developed a novel Attention Flexibility Training (AFT) based on: 1) prior research suggesting that children's attentional preference is not an adaptive or maladaptive trait per se, but rather functional depending contextual factors like the occurrence of stress (Van de Walle et al., 2017); and 2) theoretical predictions that trust in maternal support is characterized by children's capacity to flexibly shift their attention from the mother during stress to exploration in absence of stress (Main et al., 2005). We tested whether training children to adjust their attentional preference for their mother during stress versus exploration contexts increases children's trust in maternal support. Results supported the hypothesis that children's attentional flexibility can be manipulated through the AFT and that increasing attentional preference for the mother during stress contexts causally increases children's trust in her support.

The large AFT effects confirmed prior research by Lazarov et al. (2017) that a gazecontingent music reward design may be a powerful strategy to manipulate attention biases 
and should be considered in future ABM research. Adding to Lazarov et al.'s (2017) paradigm, the current study results show that it is possible to use this strategy to train individuals to focus on different stimuli depending on contextual characteristics (i.e., train attention flexibility). This is an important finding in light of the ongoing search for valid $A B M$ strategies in other research areas (e.g., MacCleod et al., 2009; Sanchez, Everaert \& Koster, 2016). The current finding contributes to the general idea that attention biases have different functions in different situations and therefore, that it is less adequate to train individuals' attentional focus exclusively toward or away from meaningful stimuli as it has been done in most previous research (Cristea et al., 2015).

Results showed that the training significantly decreased children's switch cost over time. Compared to the yoked-control condition, children in the AFT condition even learned to perform better on the trials that required switching than the trials that required to maintain the ongoing attentional strategy. In addition, the current results show that increasing children's attentional flexibility increases children's attentional focus on mother during distress. This finding is a first confirmation of Main (2000) and Bretherton's (1985) assumption that children's ability to seek maternal support during distress is linked to their ability to flexibly adjust their attentional focus on contextual demands. In light of the increasing interest on links between attachment and the attentional processing of emotionally relevant stimuli (Dykas \& Cassidy, 2011), this suggests that our innovative approach could provide an important step to better unravel the meaning and function of attachment-related attention biases.

Interestingly, the flexibility training had only a significant effect on the stress trials and not on the exploration trials because there seemed to be an overall strong preference for peers from the start of the training during exploration trials. Moreover, during the stress 
trials, children in the control condition showed increased attentional preferences for peers and decreased attention preferences for mother across the training. These findings may be important in light of the theory that secure attachment is a condition for exploration (Bowlby, 1969). These results suggest that middle childhood attention might be more strongly focused on exploration than on searching support. As this is one of the first experimental studies on the relationship between ability to seek support and exploration, more research is needed to understand this effect. This effect could be typical for the normal population sample that participated in the study. Such samples are on average more securely attached compared to children with mental health problems (Madigan et al., 2016) and therefore the sample could display a stronger inclination toward exploration. On the other hand, the large effect sizes could mean that all children are oriented toward social exploration independent of their level of trust and that attachment is a facilitator rather than a condition for exploration.

In the stress trials, the flexibility training increased children's attentional preference for their mother and decreased children's preference for peers. This suggests that the training stimulated children's support seeking during stress. This finding is in line with the growing awareness that children need to learn to seek support in response to age-specific stressors (Bosmans et al., 2017). Peer conflict is an emerging source for stress in middle childhood (Vandevivere et al., 2015) and children need to learn that mothers can also be a resource for less known stressors (Bosmans, 2016). The current paradigm seems to be able to capture the developmental task relevant for middle childhood (Vandevivere et al., 2015).

No main training effects were found on trust. However, in support of our main research question, results suggested that, only in the AFT condition, children's increased attentional preference for mother in stress conditions was linked with children's increased 
trust in maternal support. Although the number of tests we conducted requires caution for type I errors when interpreting the results, three out of four correlations with the attentional preference measures pointed to the same effect, ranging from marginal significance to significance after controlling for multiple testing. Further support for the relevance of the current results was the finding that increases in mother-focused fixation duration and fixation count indirectly linked AFT effects to increased trust. The fact that trust effects were mainly found for the mother-related attentional preference variables was in line with the developmentally-congruent finding that the improved attentional flexibility mainly reflected increased ability to focus on mother during stress. Moreover, results are in line with prior evidence found by De Winter et al. (2017) that increasing children's secure information processing biases causally increases children's trust. Adding to the latter study, our study clearly shows that this causal role is not restricted to interpretation biases, but can also be found for early-stage attention biases. Given the central role of attentional biases in the cognitive processing of information, the current findings provide stronger support for the hypothesis that information processing biases have a causal effect on the content of attachment-related expectations (Bowlby, 1969).

These results could be considered surprising in light of the small number of training trials (32) that affected changes in trust. However, the fact that the manipulation changed the attentional preference and the switching cost with large effect sizes suggests that the manipulation itself was very effective. This might be because of the use of music the children knew well. If the music gets interrupted this induces immediate frustration because it deprives the listener from the anticipated next notes/words. Therefore, adjusting attentional strategies is highly reinforced by the rewarding effect of hearing the next lines of the music and by the avoidance of this frustration. This strong effect has also been observed 
by Lazarov et al. (2017). This way, the manipulation procedure can help explain why such a brief manipulation could already change levels in trust.

Instead, in the current study, we found no support for the hypothesis that pretraining attachment anxiety or attachment avoidance moderated the training effects at the level of the attentional bias indices nor at the level of training-induced changes in trust. The amount of interaction analyses we conducted significantly increased the risk for Type I error. Nevertheless, the fact that none of the analyses revealed significant interactions argues against such a moderating effect. This conclusion is further supported by the small effect sizes. They are an indication that testing these effects with larger samples might not substantially change the conclusions. It remains important that future research tests these interactions with other types of measures to identify attachment anxiety and avoidance such as observations and interviews. However, for now, the data suggests that pre-existing insecure attachment representations have no effect on the manipulation outcomes.

When evaluating the relevance of these findings for attachment theory, important limitations need to be considered. This is the first time the current study's AFT paradigm has been used. Although the gaze-contingent reward strategy's validity has been demonstrated before in the study of Lazarov et al. (2017), more research, aimed at replicating the attachment-related effects is needed. Moreover, although the current findings seem to be a promising proof-of-principle, more research is needed with other control conditions, to test, for example, the extent to which the observed effect is driven by the fact that the mother is the familiar person (i.e., whether similar effects would be observable when using other stimuli to train attention flexibility, such as father or teachers' pictures, instead of mother's pictures). Note, however, that failure to proof that the effect is specific for mother does not reduce the relevance of the findings. Also outside of the home, more securely attached 
children are more able to rely on other adults for support, like teachers (Verschueren, 2015). Additionally, as a proof-of-principle, it is promising that the findings are in line with what De Winter et al. $(2017 ; 2018)$ found using a similar paradigm that was focused on changing verbally-explicit interpretation biases. Furthermore, it would be interesting to see whether the observed effects generalize to indices of attention flexibility that are measured outside of the training, using further attention transfer tasks. Moreover, the outcome of the training in our study is restricted to the evaluation of self-reported attachment measures. In spite of the growing consensus that these measures are of relevance to study middle childhood attachment (Bosmans \& Kerns, 2015), it would be interesting to see whether the results replicate with other measurement strategies such as interviews or observation.

In spite of these limitations, the current results suggest that children's ability to flexibly orient their attention to mother in times of distress can causally affect children's trust in the availability of their mother. The effect of the manipulation on children's attentional performance and on self-reported trust was not moderated by pre-manipulation levels of trait insecure attachment. This finding is to some extent in line with contemporary theory on the role of information processing biases (MacLeod et al., 2009) as it suggests that the biases affect the expectations and not vice versa. Moreover, the fact that insecure attachment styles did not moderate this effect is in line with accumulating middle childhood research showing that attachment traits are still under development (Waters et al., 2019) and are not yet crystallized to the point where they reduce the immediate impact of ad hoc attachment experiences (Vandevivere et al., 2018; Bosmans et al., 2014). If the current pattern of findings could be replicated, this could suggest that attachment-related information processing biases play a much more critical role in attachment development than previously assumed. If they indeed outweigh the effect of pre-existing attachment- 
related expectation patterns, they might not only explain attachment (in)stability as has always been assumed by attachment theory (Bowlby, 1969), they might even at least partly explain the mechanisms through which children construct attachment representations based on learning experiences. This way, the current findings might contribute to unraveling one of the important black boxes of attachment theory.

Future research should focus on the duration of these effects and whether they can be found in clinical populations. If such studies further support the causal effect of attachment-related attention flexibility on children's attachment security over time, highly promising venues for novel clinical interventions could be extracted from this innovative AFT approach. Given the often small effect sizes of existing treatments of child and adolescent psychopathology, adding AFT to existing parent trainings could render such training procedures more effective and help to restore insecure attachment relationships (Bosmans, 2016; Verhees, Ceulemans, \& Bosmans, 2019). 


\section{References}

Bosmans, G. (2016). Cognitive behavior therapy for children and adolescents: Can attachment theory contribute to its efficacy? Clinical Child and Family Psychology Review, 19, 310-328. doi: 10.1007/s10567-016-0212-3

Bosmans, G., \& Kerns, K. A. (2015). Attachment in Middle Childhood: Theoretical Advances and New Directions in an Emerging field. New Directions for Child and Adolescent Development, 148, 1-14. doi:10.1002/cad.20100

Bosmans, G., Van de Walle, M., Goossens, L., \& Ceulemans, E. (2014). (In) variability of attachment in middle childhood: Secure base script evidence in diary data. Behaviour Change, 31, 225-242. doi: 10.1017/bec.2014.18

Bosmans, G., Koster, E. H. W., Vandevivere, E., Braet, C., \& De Raedt, R. (2013). Young adolescent's confidence in maternal support: Attentional bias moderates the link between attachment-related expectations and behavioral problems. Cognitive Therapy and Research, 37, 829-839. http://doi.org/10.1007/s10608-013-9526-3

Bosmans, G., Goldblum, E., Braet, C., Van de Walle, M., Heylen ,J., Bijttebier, P., Santens, T., Koster, E., De Raedt, R. (2017). Children's attentional breadth around mother: Comparing stimulus-driven versus cognitively controlled processes. Social Development. Bowlby, J. (1969). Attachment and loss: Vol. 1. Attachment. New York, NY: Basic Books.

Brenning, K. M., Van Petegem, S., Vanhalst, J., \& Soenens, B. (2014). The psychometric qualities of a short version of the Experiences in Close Relationships Scale - Revised Child version. Personality and Individual Differences, 68, 118-123. doi:10.1016/j.paid.2014.04.005

Bretherton, I. (1985). Attachment theory: Retrospect and prospect. Monographs of the Society for Research in Child Development, 3-35. doi: 10.2307/3333824 
Cassidy, J. (1994). Emotion regulation: Influences of attachment relationships. Monographs of the Society for Research in Child Development, 59(2/3, Serial No.240), 228-249. http://doi.org/10.2307/1166148

Cristea, I.A., Kok, R.N., \& Cuijpers, P. (2015). The efficacy of cognitive bias modification interventions in anxiety and depression: A meta-analysis. British Journal of Psychiatry, 206, 7-16.

Del Giudice, M. (2015). Attachment in Middle Childhood: An Evolutionary-Developmental Perspective. New Directions for Child and Adolescent Development, 148, 15-30. doi:10.1002/cad.20100

Derryberry, D., \& Tucker, D. M. (1994). Motivating the focus of attention. In P. M. Niedenthal \& S. Kitayama (Eds.), The heart's eye: Emotional influences in perception and attention (pp. 167-196). San Diego, CA: Academic Press.

De Winter, S., Bosmans, G., \& Salemink, E. (2017). Brief Report: Exploring the causal effect of interpretation bias on attachment expectations. Child Development, 88, 131-140.

De Winter S., Salemink E., Bosmans G. (2018). Interpretation Bias in Middle Childhood Attachment: Causal Effects on Attachment Memories and Scripts. Behaviour Research and Therapy, 102, 16-24.

Dujardin, A., Santens, T., Braet, C., De Raedt, R., Vos, P., Maes, B., \& Bosmans, G. (2016). Middle childhood support-seeking behavior during stress: Links with self-reported attachment and future depressive symptoms. Child Development, 87, pp. 326-340. doi: 10.1111/cdev.12491.

Dykas, M. J., \& Cassidy, J. (2011). Attachment and the processing of social information across the life span: Theory and evidence. Psychological Bulletin, 137, 19-46. http://doi.org/10.1037/a0021367 
Hayes, A. F. (2012). PROCESS: A versatile computational tool for observed variable mediation, moderation, and conditional process modeling [White paper]. Retrieved from http://www.afhayes.com/public/process2012.pdf

Heeren, A., Mogoas, e, C., Philippot, P., \& McNally, R. J. (2015). Attention bias modification for social anxiety: a systematic review and meta-analysis. Clinical Psychology Review, 40, 76-90.

Kirsh, S. J., \& Cassidy, J. (1997). Pre-schoolers' attention to and memory for attachmentrelevant information. Child Development, 68, 1143-1153.

Lakens, D. (2013). Calculating and reporting effect sizes to facilitate cumulative science: A practical primer for t-tests and ANOVAs. Frontiers in Psychology, 4, 863. doi:10.3389/fpsyg.2013.00863.

Lazarov, A., Pine, D. S., \& Bar-Haim, Y. (2017). Gaze-contingent music reward therapy for social anxiety disorder: A randomized controlled trial. American Journal of Psychiatry, $174,649-656$.

MacLeod, C., Koster, E. H. W., \& Fox, E. (2009). Whither cognitive bias modification research? Commentary on the special section articles. Journal of Abnormal Psychology, 118, 89-99. http://dx.doi.org/10.1037/a0014878.

Madigan, S. et al., (2016). Representational and questionnaire measures of attachment: A meta-analysis of relations to child internalizing and externalizing problems. Psychological Bulletin, 142, pp.367-299.

Main, M. (2000). The organized categories of infant, child, and adult attachment: flexible vs. inflexible attention under attachment-related stress. Journal of American Psychoanalytical Association, 48, 1055-1096. doi:10.1177/00030651000480041801

Main, M., Hesse, E., \& Kaplan, N. (2005). Predictability of attachment behavior and representational processes at 1, 6, and 19 years of age. In K. E. Grossmann, K. 
Grossmann, E. Waters (Eds.), Attachment from infancy to adulthood: The major longitudinal studies (pp.245-304). London: Guilford press.

Ridenour, T. A., Greenberg, M. T., \& Cook, E. T. (2006). Structure and validity of People In My Life: A self-report measure of attachment in late childhood. Journal of Youth and Adolescence, 35, 1037-1053. http://doi.org/10.1007/s10964-006-9070-5

Sanchez, A., Everaert, J., De Putter, L., Mueller, S., \& Koster, E. H. W. (2015). Life is ... great! Emotional attention during instructed and uninstructed ambiguity resolution in relation to depressive symptoms. Biological Psychology, 109, 67-72. doi:10.1016/i.biopsycho.2015.04.007

Sanchez, A., Everaert, J., Koster, E.H.W. (2016).Attention training through gaze-contingent feedback: effects on reappraisal and negative emotions. Emotion, 16, 1074-1085. doi:10.1037/emo0000198

Thompson, R. A. (2008). Attachment-related mental representations: Introduction to the special issue. Attachment \& Human Development, 10, 347-358. http://doi.org/10.1080/14616730802461334

Vandevivere, E., Braet, C., \& Bosmans, G. (2015). Under which Conditions do Early Adolescents Need Maternal Support? Journal of Early Adolescence, 35, 162-169. doi:10.1177/0272431614529364

Vandevivere, E., Bosmans, G., Roels, S., Dujardin, A., \& Braet C. (2018). State Trust in Middle Childhood : An Experimental Manipulation of Maternal Support. Journal of Child and Family Studies, 1252-1263. doi:10.1007/s10826-017-0954-7

Van de Walle, M., Bijttebier, P., De Raedt, R., \& Bosmans, G. (2017). Repetitive thinking about mother during distress moderates the link between children's attentional breadth around the mother and depressive symptoms in middle childhood. Behaviour 
Research and Therapy, 90, 137-146. http://dx.doi.org/10.1016/j.brat.2016.12.002 Verschueren, K. (2015). Middle childhood teacher-child relationships: Insights from an attachment perspective and remaining challenges. In G. Bosmans \& K. A. Kerns (Eds.), Attachment in middle childhood: Theoretical advances and new directions in an emerging field. New Directions for Child and Adolescent Development, 148, 77-91.

Waters, T.E.A., Facompré, C.R., Dujardin, A., Van de Walle, M., Verhees, M., Bodner, N., Boldt, L.J., \& Bosmans, G. (2019). Taxometric analysis of secure base script knowledge in middle childhood reveals categorical latent structure. Child Development. DOI: 10.1111/cdev.13229.

Zimmermann, P., \& Iwanski, A. (2015). Attachment in middle childhood: Associations with information processing. In G. Bosmans \& K. A. Kerns (Eds.), Attachment in middle childhood: Theoretical advances and new directions in an emerging field. New Directions for Child and Adolescent Development, 148, 47-61. 
Table 1: Repeated measures ANOVA predicting Fixation Duration and Fixation Count changes

\begin{tabular}{|c|c|c|c|c|}
\hline & \multicolumn{2}{|c|}{ Fixation Duration } & \multicolumn{2}{|c|}{ Fixation Count } \\
\hline & $F$ & $\eta^{2}$ & $F$ & $\eta^{2}$ \\
\hline Time & .00 & .00 & .48 & .01 \\
\hline Trial Type & 1.28 & .02 & $14.95^{* * *}$ & .15 \\
\hline Target Stimulus & $13.23^{* * *}$ & .14 & $47.99 * * *$ & .37 \\
\hline Time $X$ Condition & .27 & .00 & 1.33 & .02 \\
\hline Trial Type X Condition & .53 & .00 & 3.34 & .04 \\
\hline Target Stimulus X Condition & 3.89 & .05 & 3.75 & .04 \\
\hline Time $X$ Trial Type & 1.20 & .01 & 2.33 & .03 \\
\hline Time $\mathrm{X}$ Target Stimulus & 1.93 & .02 & 1.64 & .02 \\
\hline Trial Type X Target Stimulus & $71.78 * * *$ & .47 & $70.57 * * *$ & .46 \\
\hline Time $X$ Trial Type $X$ Condition & .00 & .00 & .24 & .00 \\
\hline Time $\mathrm{X}$ Target Stimulus $\mathrm{X}$ Condition & $15.19 * * *$ & .16 & $9.32 * *$ & .10 \\
\hline Trial Type X Target Stimulus X Condition & $63.36 * * *$ & .44 & $48.67^{* * *}$ & .37 \\
\hline Time X Trial Type X Target Stimulus & $16.68^{* * *}$ & .17 & $7.39 * *$ & .08 \\
\hline Time $X$ Trial Type $X$ Target Stimulus $X$ Condition & $19.03 * * *$ & .19 & $18.90 * * *$ & .19 \\
\hline
\end{tabular}


Table 2: correlations between standardized residual change scores related to stress trials for the AFT condition (below the diagonal) and the control condition (above the diagonal) separately

\begin{tabular}{lllllll}
\hline & \multicolumn{1}{c}{1} & \multicolumn{1}{c}{2} & 3 & 4 & 5 & 6 \\
\hline 1. Trust & 1 & .17 & .23 & .05 & -.07 & -.12 \\
2. Fix Duration Mother & $.29^{+}$ & 1 & $-.56^{* * *}$ & $.37^{*}$ & $-.53^{* * *}$ & .20 \\
3. Fix Duration Peers & $-.36^{*}$ & $.81^{* * *}$ & 1 & $-.29 *$ & $.61^{* * *}$ & -.07 \\
4. Fix Count Mother & $.38^{*}$ & $.38^{*}$ & $-.38^{*}$ & 1 & -.18 & -.10 \\
5. Fix Count Peers & -.17 & $-.68^{* * *}$ & $.79 * * *$ & -.12 & 1 & .13 \\
6. Switch Cost & .22 & $.56^{* * *}$ & $-.53^{* * *}$ & .21 & $-.36^{*}$ & 1 \\
\hline
\end{tabular}

Note: ${ }^{+} p<.10 ;{ }^{*} p<.05 ;{ }^{* *} p<.01{ }^{* * *} p<.001$

Figure 1: Stress trial 


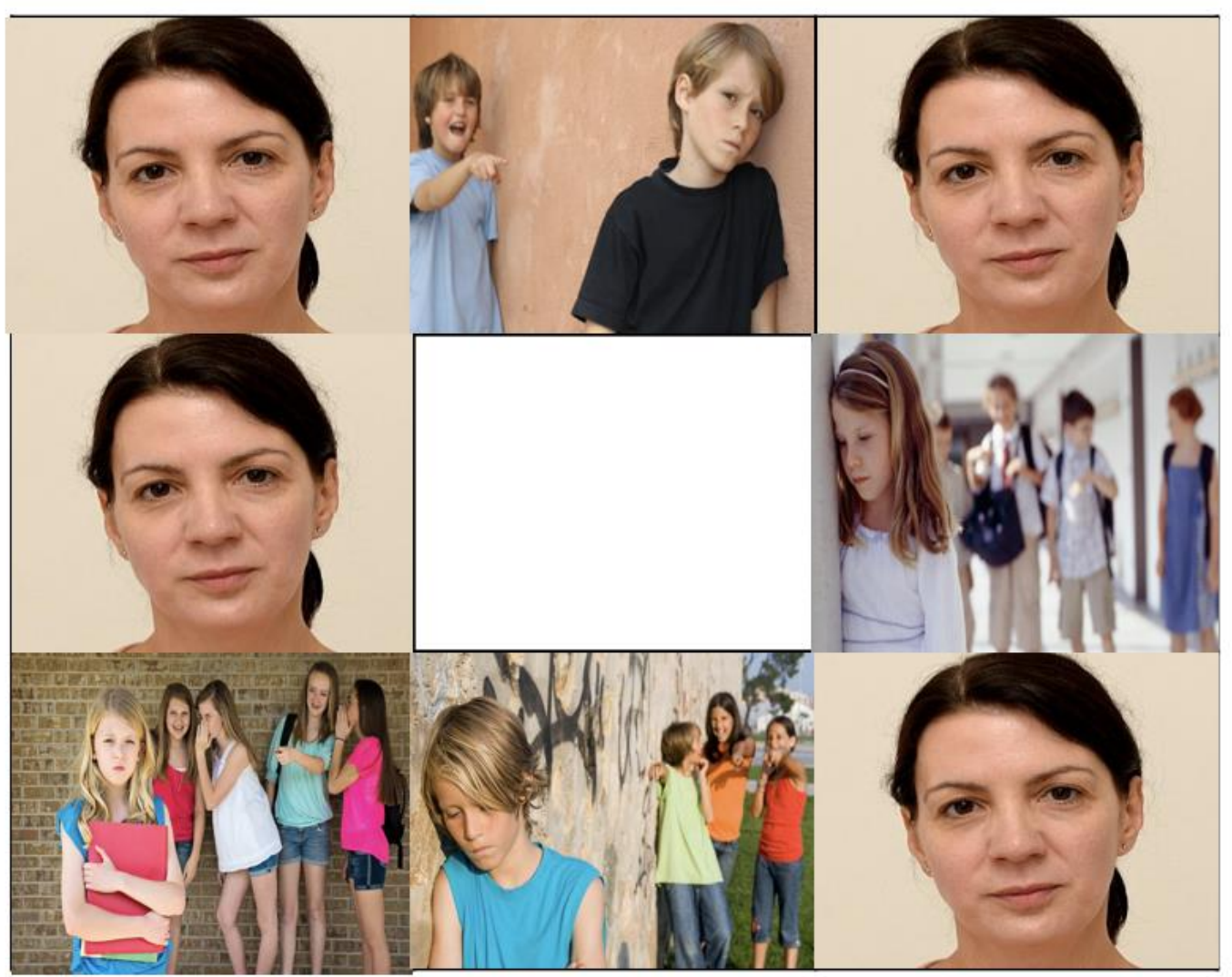

Figure 2: Exploration trial 


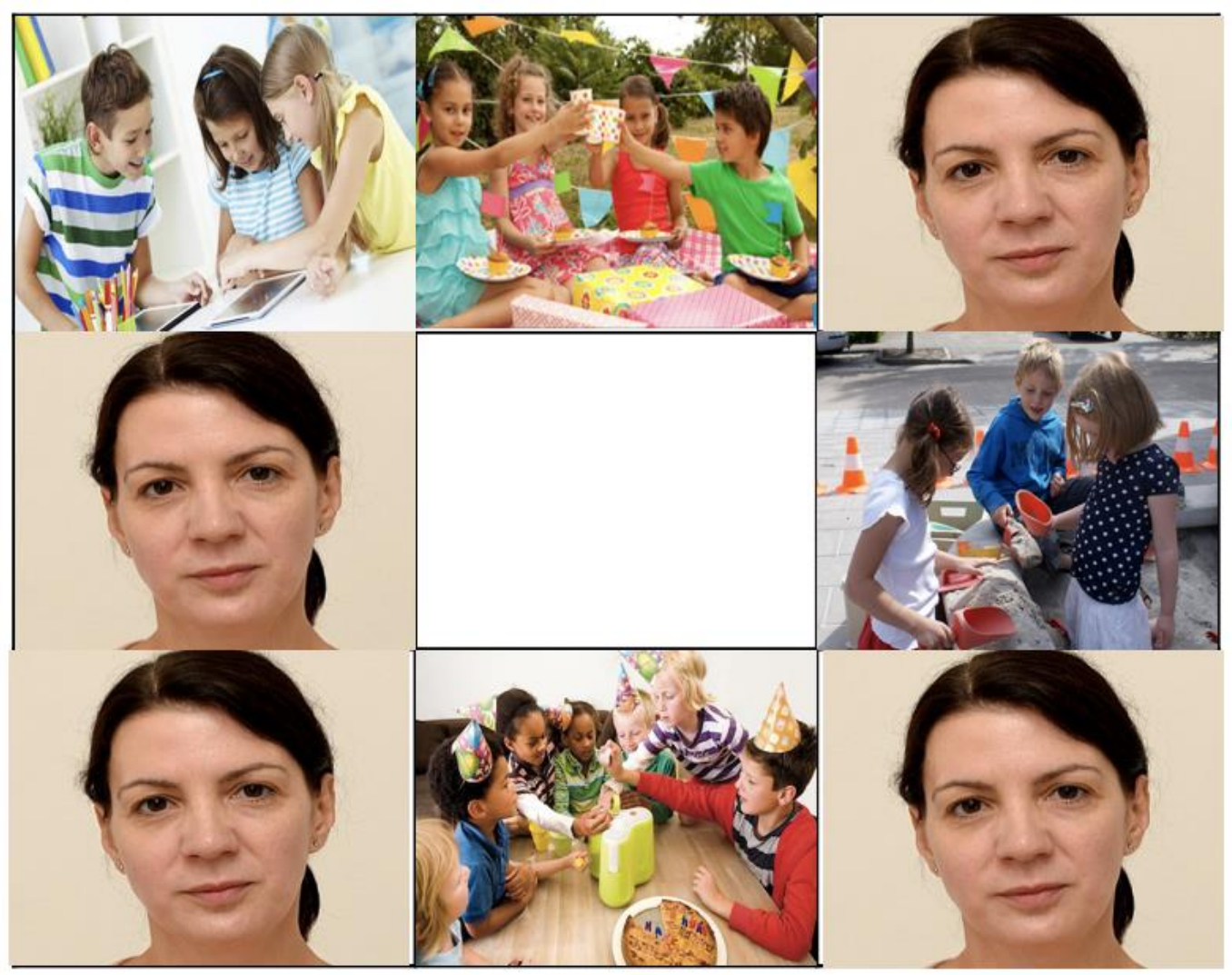


Figure 3: Changes in attentional preference for mother and peers for the entire fixation duration during the stress trials

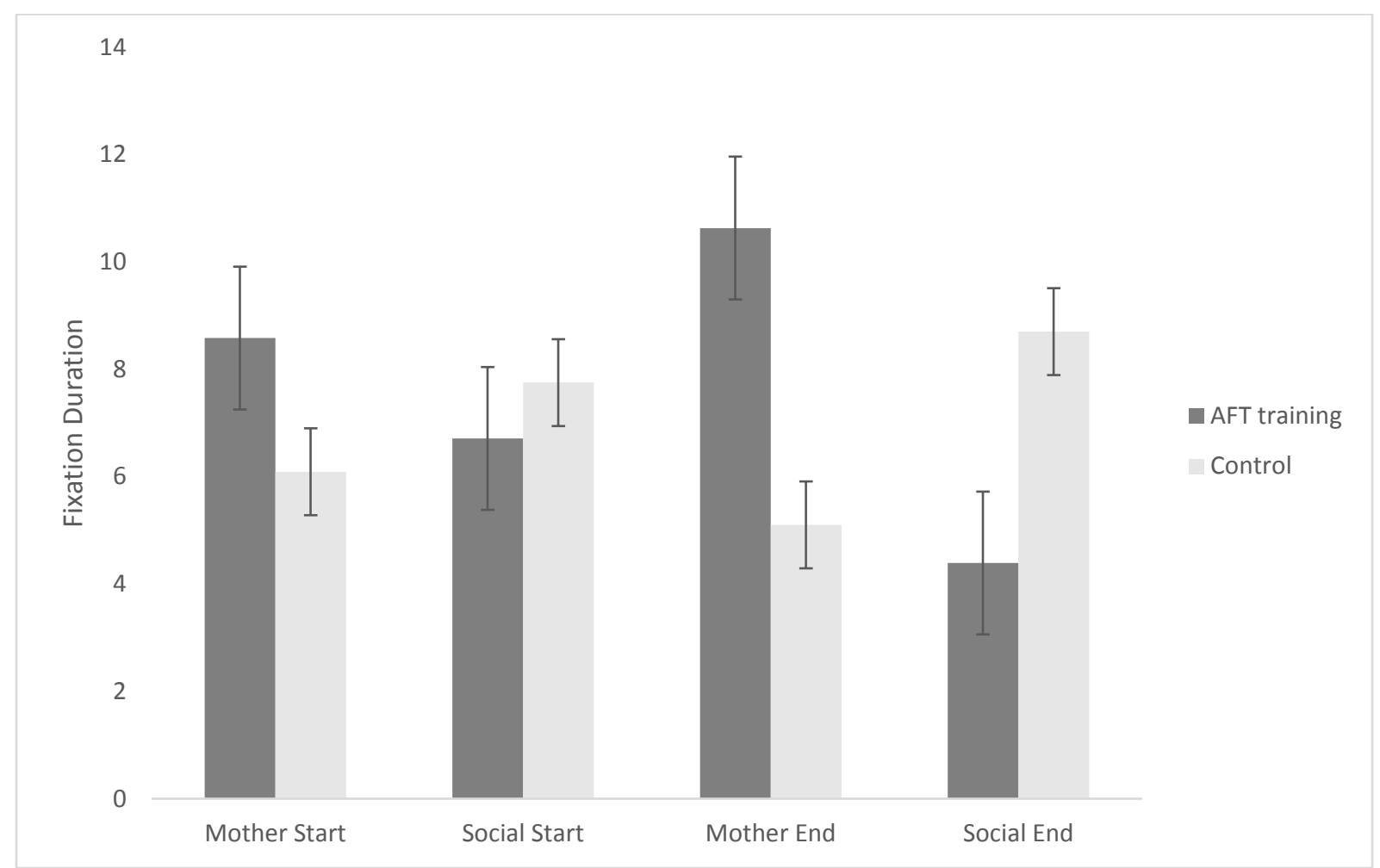


Figure 4: Changes in attentional preference for mother and peers for total fixation count during the stress trials

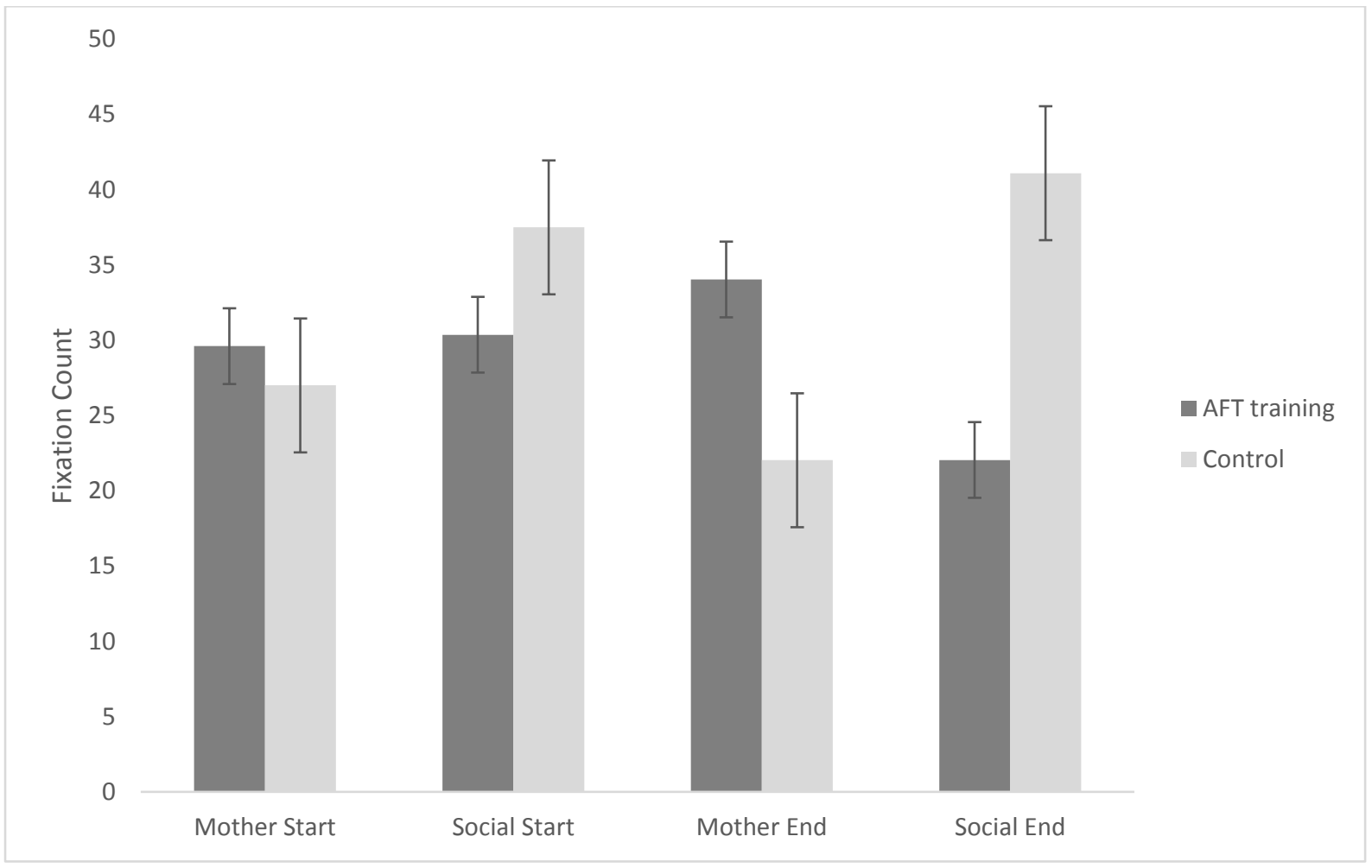


Figure 5: Change in switch cost over time

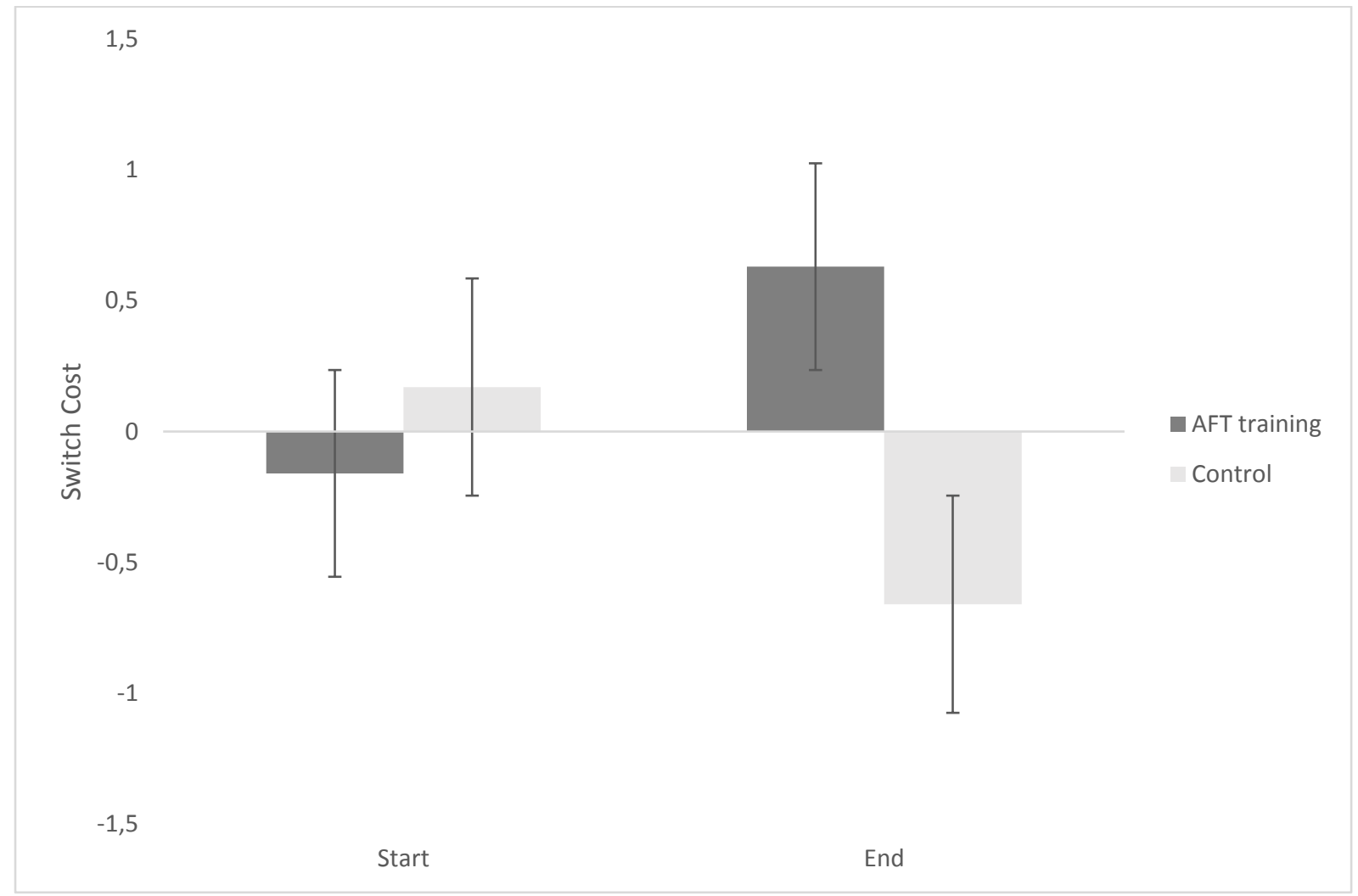

